\title{
Post-seismic reloading and temporal clustering on a single fault
}

\author{
Christopher J. DiCaprio, ${ }^{1}$ Mark Simons, ${ }^{1}$ Shelley J. Kenner ${ }^{2}$ and Charles A. Williams ${ }^{3}$ \\ ${ }^{1}$ Seismological Laboratory, California Institute of Technology, Pasadena, CA, USA. E-mail: dicaprio@gps.caltech.edu \\ ${ }^{2}$ Department of Earth and Environmental Sciences, University of Kentucky, Lexington, KY, USA \\ ${ }^{3}$ Department of Earth and Environmental Sciences, Rensselaer Polytechnic Institute, Troy, NY, USA
}

Accepted 2007 September 19. Received 2007 July 14; in original form 2007 March 12

\begin{abstract}
SUMMAR Y
Geological studies show evidence for temporal clustering of large earthquakes on individual fault systems. Since post-seismic deformation due to the inelastic rheology of the lithosphere may result in a variable loading rate on a fault throughout the interseismic period, it is reasonable to expect that the rheology of the non-seismogenic lower crust and mantle lithosphere may play a role in controlling earthquake recurrence times. We study this phenomenon using a 2-D, finite element method continuum model of the lithosphere containing a single strike-slip fault. This model builds on a previous study using a 1-D spring-dashpot-slider analogue of a single fault system to study the role of Maxwell viscoelastic relaxation in producing non-periodic earthquakes. In our 2-D model, the seismogenic portion of the fault slips when a predetermined yield stress is exceeded; stress accumulated on the seismogenic fault is shed to the viscoelastic layers below and recycled back to the seismogenic fault through viscoelastic relaxation. We find that random variation of the fault yield stress from one earthquake to the next can cause the earthquake sequence to be clustered; the amount of clustering depends on a non-dimensional number, $W$, called the Wallace number defined as the standard deviation of the randomly varied fault yield stress divided by the effective viscosity of the system times the tectonic loading rate. A new clustering metric based on the bimodal distribution of interseismic intervals allows us to investigate clustering behaviour of systems over a wide range of model parameters and those with multiple viscoelastic layers. For models with $W \gtrsim 1$ clustering increases with increasing $W$, while those with $W \lesssim 1$ are unclustered, or quasi-periodic.
\end{abstract}

Key words: Seismic cycle; Statistical seismology; Rheology: crust and lithosphere.

\section{INTRODUCTION}

Several geological studies show evidence of temporal clustering of large earthquakes on individual faults. Wallace (1987) finds changes in slip rates along range front faults in the Great Basin over the past $10 \mathrm{Myr}$; in addition, subprovinces of the Great Basin tend to have clusters of earthquakes while other areas are inactive. Similarly, Friedrich et al. (2003) find that the faults bounding the Wasatch range exhibit temporal clustering. A palaeoseismic study of the Dead Sea transform shows strong temporal clustering of events over the past $50000 \mathrm{yr}$ (Marco et al. 1996; Begin et al. 2005). Trenching studies on the Carrizo Plain segment of the San Andreas Fault (Grant \& Sieh 1994) and near Wrightwood, California (Weldon et al. 2004) show that earthquakes on a single fault segment can cluster in time.

Many modelling studies have previously addressed clustering of large earthquakes. Ben-Zion et al. (1999) used a model of an upper crust with damage rheology overlying a viscoelastic substrate to model multiple evolving fault systems that exhibit clustering behaviour. Lyakhovsky et al. (2001) found similar results from a model of a single strike-slip fault system. In a study of changing fault slip rates due to changing fault friction Chery \& Vernant (2006) showed that an elastically weak lithosphere contributes to large fluctuations in fault slip rate; large fault rate variations were present in models with a strain weakening fault with a short weakening time relative to the tectonic loading rate. Chery et al. (2001) showed that the viscoelastic post-seismic deformation from one fault could bring a neighbouring fault closer to failure. The two parallel strike-slip fault system exhibited temporal clustering behaviour under certain values of low crustal viscosity and tectonic strain rate. Similar results have been found for a model of the San Andreas fault that has two seismogenic segments on a single strike-slip fault separated by an aseismically slipping segment (Lynch et al. 2003). Generally, it appears that any rheology with memory (e.g. viscoelastic and damage rheologies) is susceptible to clustering behaviour. Here we focus on viscoelastic rheologies since they are the most commonly adopted constitutive laws in the lithospheric modelling community.

After an earthquake, post-seismic viscoelastic deformation rates can be on the same scale as the tectonic loading rates (e.g. Savage \& Prescott 1978; Kenner \& Segall 2000; Meade \& Hager 2004). We therefore, expect that recycling of stress via post-seismic relaxation 
may play a role in the timing of the next earthquake on a single fault. Kenner \& Simons (2005) (henceforth referred to as KS05) developed a 1-D spring-dashpot-slider model as an analogue to a layered viscoelastic lithosphere with a single strike-slip fault to study temporal earthquake clustering due to reloading of the seismogenic fault by post-seismic relaxation. Here, we investigate earthquake clustering due to viscoelastic relaxation using a 2-D, finite element method (FEM) continuum model of the lithosphere containing a single infinitely long strike-slip fault. Generally, we wish to use a more physically grounded model, since the 1-D model has no inherent length scale. Multiple viscoelastic layers are represented by $\mathrm{KS} 05$ as viscoelastic spring-and-dashpot elements connected in parallel; in this case, the clustering behaviour of a system with multiple viscoelastic elements is identical to that of a system with a single viscoelastic element with an effective viscosity that is the arithmetic mean of all viscosities. However, in a 2-D model, the viscoelastic mantle can only communicate with the elastic upper crust through the lower crust; we would therefore, expect more complex behaviour. The temporal clustering behaviour of the system is expected to be a function of the geometry (thickness) of the layers as well as their viscosities.

KS05 show that a viscoelastic feedback system in the lithosphere can produce clustered earthquake sequences on a single fault in their 1-D model. Stress transferred coseismically from the elastic element to Maxwell viscoelastic elements is recycled through viscoelastic relaxation back to the elastic element. The system is loaded by a constant velocity boundary condition representing a steady-state tectonic load. The fault is modelled by a slider block, allowed to slip with zero kinetic friction when a specified yield force is exceeded. Elasticity in the seismogenic layer is provided by a spring element and Maxwell viscoelastic behaviour is provided by spring and dashpot (damper) elements connected in series. The seismogenic element and all viscoelastic elements are connected in parallel to represent various rheological layers in the lithosphere.

KS05 demonstrate that a viscoelastic feedback system can have clustered earthquake sequences when the yield stress of the block slider changes by a small random amount from one earthquake to the next due to normally distributed noise added to the yield stress. They find that the degree of clustering is controlled by a non-dimensional number $W_{\mathrm{KS} 05}$, called the Wallace number after Robert Wallace who demonstrated the existence of clustered earthquakes in the Basin and Range province (Wallace 1987).

$W_{\mathrm{KS} 05}=\frac{\overline{\Delta \tau}}{\overline{\epsilon_{o}} \eta_{\mathrm{eff}}}$,

where $\overline{\Delta \tau}$ is the average yield stress for all earthquakes, $\dot{\epsilon}_{o}$ is the applied strain rate, and $\eta_{\text {eff }}$ is the effective viscosity of the system (see Table 1 for a list of nomenclature used in the text). $W_{\mathrm{KS} 05}$, as originally defined, has many problems that we address in what follows. We develop a revised definition of the Wallace number that has sensitivity to the amount of noise in the system. The KS05 subscript is meant to distinguish their incorrect formulation of the Wallace number, from the reformulated one presented here.

We also propose a new clustering metric, which is insensitive to the level noise in the yield stress and does not saturate for highly clustered systems, in contrast to the one used by KS05. The metric relies on the bimodality of earthquake recurrence times in clustered systems. As an aside, success of this new clustering metric suggests a need to reconsider the currently adopted earthquake recurrence models that assume unimodal distributions.
Table 1. Definition of notation used in text.

\begin{tabular}{ll}
\hline$W_{\mathrm{KS} 05}$ & Wallace number defined by KS05, \\
$W$ & New Wallace number \\
$C_{v}$ & Coefficient of variation, old clustering metric \\
$B_{\mathrm{c}}$ & New clustering metric based on distribution of $\log _{10}\left(T^{\mathrm{eq}}\right)$ \\
$\overline{\Delta \tau}$ & Average earthquake yield stress \\
$\sigma_{\Delta \tau}$ & Standard deviation of earthquake yield stress \\
$N$ & $\sigma_{\Delta \tau} / \overline{\Delta \tau}$ \\
$\dot{\epsilon_{o}}$ & Applied strain rate \\
$\eta_{\mathrm{eff}}$ & Effective viscosity of the 1-D system used by KS05 \\
$\eta_{i}$ & Viscosity of $i$ th layer \\
$G_{i}$ & Shear modulus of $i$ th layer \\
$T^{\mathrm{eq}}$ & Average interseismic time \\
$\overline{\Delta \epsilon}$ & Average seismic strain drop \\
$T^{h \mathrm{~s}}$ & Characteristic relaxation time of an elastic layer over a \\
& viscoelastic halfspace \\
\hline
\end{tabular}

\section{METHODS}

\subsection{Lithospheric model}

We study an antiplane, 2-D, continuum model of the lithosphere containing an infinite strike-slip fault. Two different model types are tested: one with an elastic layer over a viscoelastic layer (referred to as the two-layer model) and one with an elastic layer over two viscoelastic layers (referred to as the three-layer model). The elastic parameters are the same for all rheological layers. A single vertical, strike-slip fault extends through the entire elastic layer and penetrates $2 \mathrm{~km}$ into the viscoelastic layer. Taking advantage of symmetry, we model only one side of the fault system. The model is driven at a constant velocity, $v_{p}$, along the entire right edge (making the average slip rate for the entire fault system $2 v_{p}$ ). The mesh is $1004 \mathrm{~km}$ in horizontal extent and $204 \mathrm{~km}$ in vertical extent. The top and bottom boundaries of the model are free surfaces (Fig. 1).

The coseismic fault is locked until it reaches a specified yield stress. At this point the fault is allowed to slide freely for one, nearly instantaneous, coseismic time step, resulting in complete stress drop. We have used log-normal noise to vary the yield stress from one earthquake to the next, with standard deviation $\sigma_{\Delta \tau}$. The ratio of the standard deviation to the average of the fault yield stress varies from 1 to 10 per cent. We make no claim that log-normal noise is the most appropriate distribution to model a natural system; the appropriate distribution of noise is unknown. A log-normal noise distribution is chosen because it doesn't allow the fault yield stress to become negative which is physically unreasonable. In this study, we are concerned only with characteristic earthquakes on a particular fault (e.g. Schwartz \& Coppersmith 1984) and not with modelling a magnitude frequency distribution. Therefore, in the absence of added noise, every earthquake in our model is the same size; when noise is introduced to the fault yield stress the size of the earthquakes will vary with the yield stress as there is complete stress drop for every earthquake.

We use the quasi-static, finite element code Tecton (Melosh \& Raefsky 1980; Williams \& Wadge 2000) to model the response of a viscoelastic lithosphere to an earthquake. We take advantage of the linear nature of the system by using a spatio-temporal Green's function approach to calculating a sequence of earthquakes. The finite element code is used to calculate the system response to a single earthquake which is then taken to be a spatio-temporal Green's function used to create a series of earthquakes by summing them with the appropriate amplitude scaling and time-shift for the size and timing of each earthquake. This approach allows the rapid calculation 


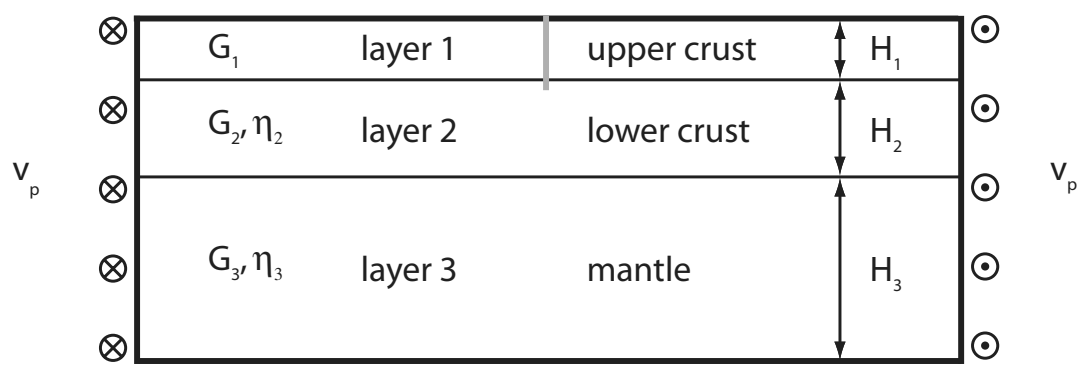

Figure 1. Model of infinite, antiplane, vertical, strike-slip fault. The upper crust is purely elastic, while the lower crust and mantle layers are viscoelastic. The fault (in grey) passes through the entire elastic layer and penetrates the top $2 \mathrm{~km}$ of the viscoelastic lower crust. Tectonic loading is introduced by a constant velocity boundary condition applied to the entire far edge of the model. The fault fails when the average shear stress on the seismogenic fault reaches a predetermined yield stress. We study models with both one and two viscoelastic layers below the elastic seismogenic crust.

of long earthquake sequences suitable for statistical analysis of temporal clustering behaviour.

The model is run for several hundreds to thousands of earthquake cycles in order to gather reliable clustering statistics. Before a statistically meaningful earthquake sequence can be generated, the model must first be spun up to a steady state which requires running the model through several earthquake cycles to load the viscoelastic layers (the number of cycles needed for spin-up increases with decreasing $\left.W_{\mathrm{KS} 05}\right)$. In the case where no noise is added to the fault yield stress, spin up is achieved when the average interseismic stresses are approximately constant from one earthquake to the next (KS05; Hetland \& Hager 2006a). An earthquake sequence with random variation in the fault yield stress is considered to be spun up when the average interseismic stress over several earthquake cycles is constant. We use the integrated elastic potential, $U$, as a metric for the amount of elastic stress stored in each rheological layer (upper crust, lower crust and upper mantle). The integrated elastic potential of the $n$th layer occupying volume $V_{n}$ is

$$
\begin{aligned}
U_{n} & =\int_{V_{n}} \frac{1}{2}\left(\lambda \epsilon_{k k}^{e} \delta_{i j}+2 \mu \epsilon_{i j}^{e}\right) \epsilon_{i j}^{e} \mathrm{~d} V \\
& =\int_{V_{n}} \frac{1}{2}\left(\frac{1+v}{E} \tau_{i j} \tau_{i j}-\frac{v}{E} \tau_{k k}^{2}\right) \mathrm{d} V,
\end{aligned}
$$

where $\lambda$ is the Lamé modulus, $\mu$ is the shear modulus, $v$ is Poisson's ratio and $E$ is Young's modulus. $\epsilon_{i j}^{e}$ is the elastic component of strain and $\tau_{i j}$ is the stress (Chandrasekharaiah \& Debnath 1994). The integrals are performed over each material layer in the model so that $U$ is a measure of the total elastic stress stored in that layer.

\subsection{Clustering metric}

Fig. 2 compares total stress released by the fault as a function of time for clustered and unclustered (quasi-periodic) earthquake
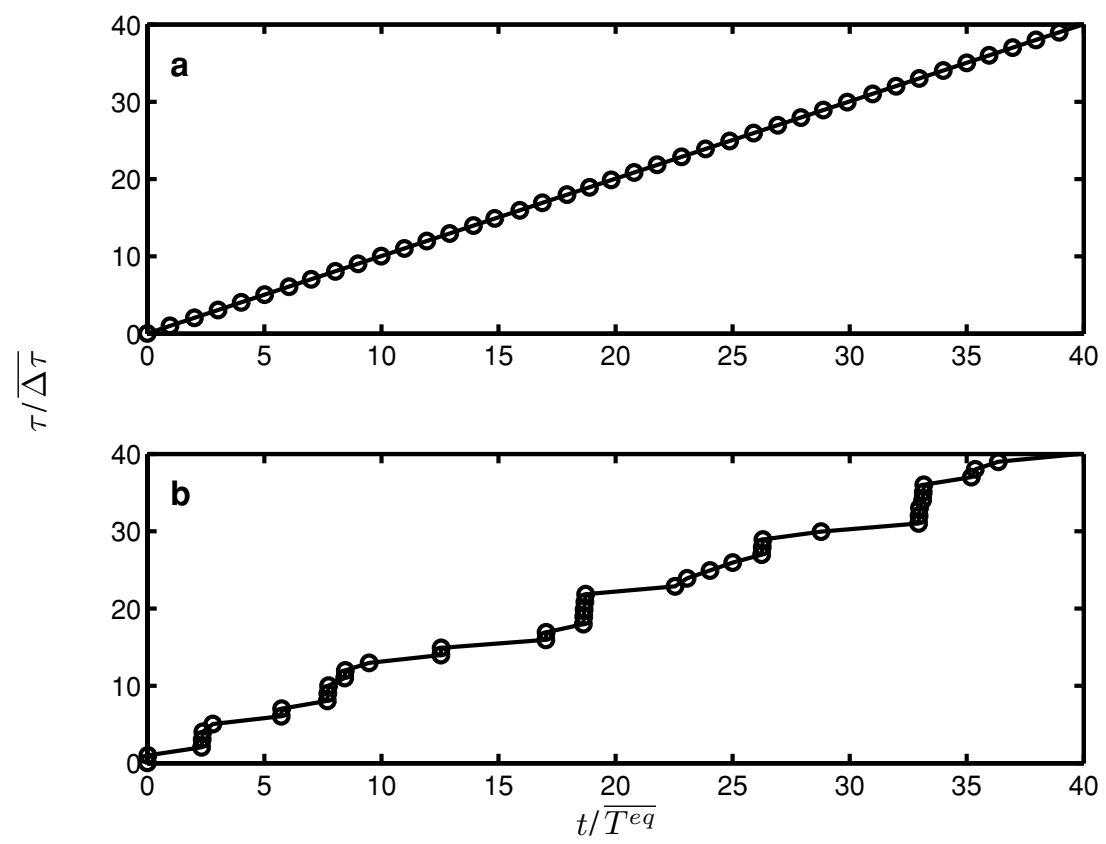

Figure 2. Cumulative stress released on the fault normalized by the mean yield stress versus normalized time. $\overline{T^{\mathrm{eq}}}$ is the mean interseismic interval. The difference between the panels is the viscosity of the lower crust (a) $W=0.14$ and (b) $W=1400$. 


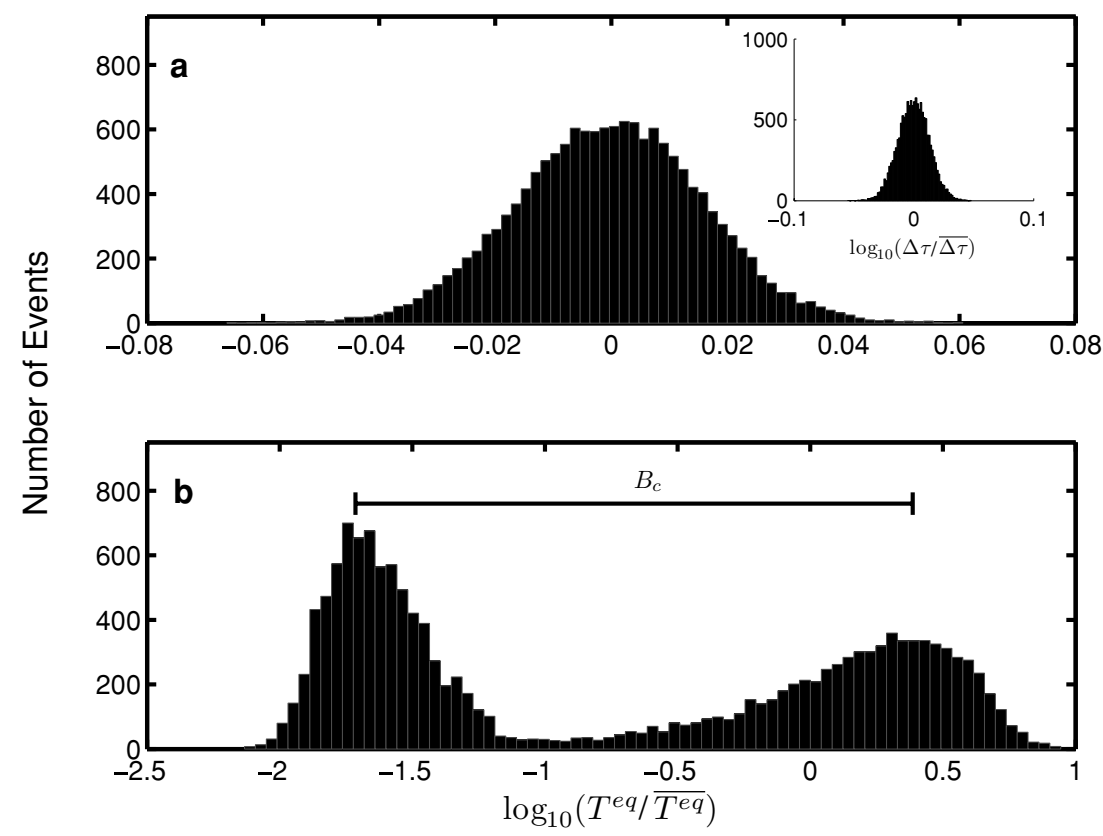

Figure 3. Distribution of normalized interseismic intervals on logarithmic scale for models with (a) $W=0.14$ and (b) $W=1400$. The distribution of fault yield stress for $\sigma_{\Delta \tau} / \overline{\Delta \tau}=0.03$ is shown in the inset.

sequences. For a quasi-periodic earthquake sequence, the distribution of interseismic intervals has the same character as the distribution of yield stresses (Fig. 3a). A clustered sequence, however, has a bimodal distribution of interseismic intervals when plotted on a logarithmic scale (Fig. 3b). The average interseismic interval is the same for both quasi-periodic and clustered sequences, as is kinematically required because they both have the same average stress drop and long-term average displacement, but the distribution of interseismic intervals is radically different.
KS05 quantify the amount of clustering using the coefficient of variation, $C_{v}$, of the interseismic times for the earthquake sequence. $C_{v}$ is the ratio of the sample standard deviation to the sample mean (Kagan \& Jackson 1991). Empirically, $C_{v}$ as a metric of clustering is problematic since it is sensitive to the amount of noise applied to the yield stress and saturates for $W_{\mathrm{Ks} 05} \gtrsim 1000$ (Fig. 4). The sensitivity of $C_{v}$ to the noise in the yield stress manifests both when the earthquake sequence is unclustered (the $C_{v}$ of the interseismic times is equal to the $C_{v}$ of the input noise) and when the earthquake

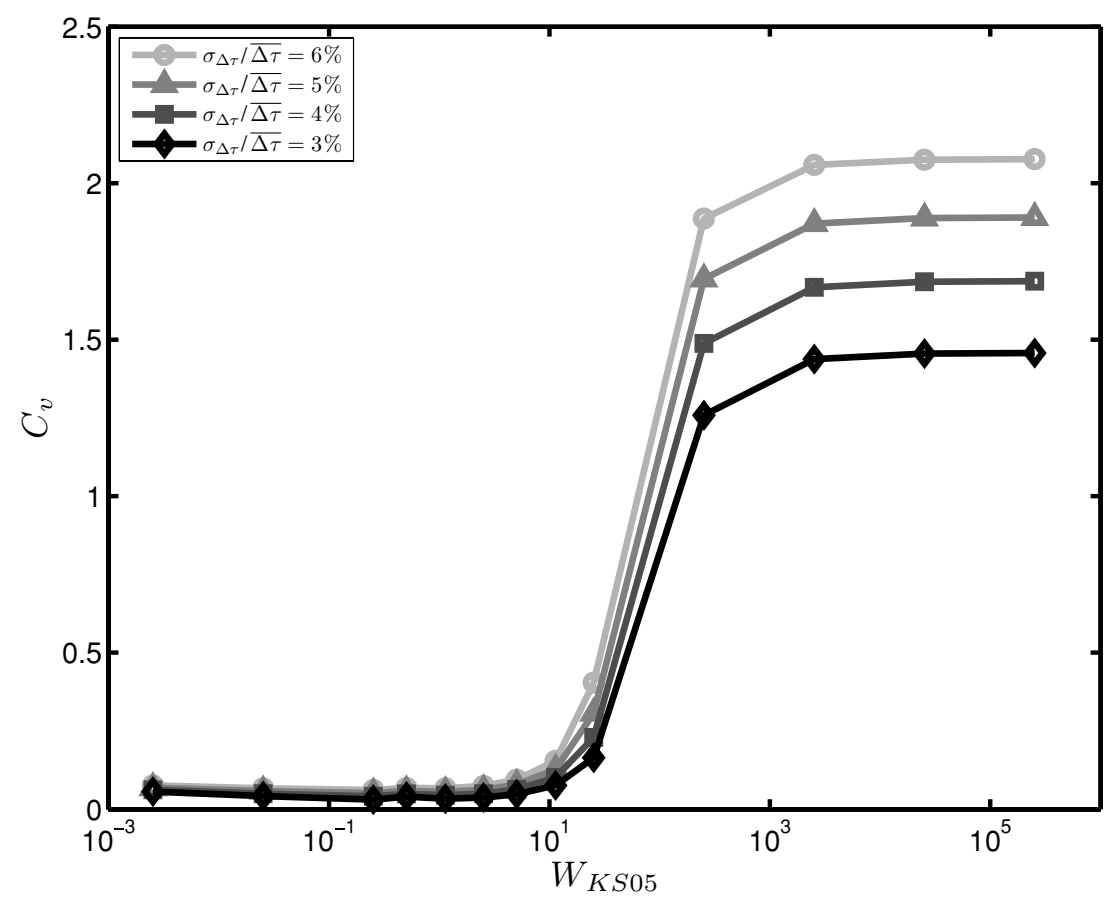

Figure 4. Amount of clustering as measured by the coefficient of variation, $C_{v}$, versus $W_{\mathrm{KS} 05}$ for two-layer models. Quasi-periodic sequences have $C_{v} \approx$ $\sigma_{\Delta \tau} / \overline{\Delta \tau}$, while larger values of $C_{v}$ indicate a clustered sequence. Note that $C_{v}$ saturates for values of $W_{\mathrm{KS} 05}>1000$. 
sequence is clustered. The distribution of the log of the interseismic intervals, $\log \left(T^{\mathrm{eq}}\right)$, reveals the bimodality of the distribution of interseismic times (Fig. 3b); $C_{v}$ is not an appropriate statistic for describing a bimodal distribution. This bimodality is not apparent on a linear scale. We propose a new metric for measuring the amount of temporal clustering of an earthquake sequence based on the distance, $B_{\mathrm{c}}$, between the two modes of the distribution of $\log \left(T^{\mathrm{eq}}\right)$ (Fig. 3b). The mode of small interseismic intervals is referred to as the 'intracluster' mode and the mode of long interseismic intervals is the 'intercluster' mode. The logarithmic distance between modes is a natural choice for quantifying the degree of clustering due to the interseismic interval being a Jeffreys quantity (Tarantola 2006).

Studies of statistical distributions of earthquake recurrence intervals and seismic hazard analyses traditionally assume that the interseismic distributions are unimodal; the success of the bimodal clustering metric, $B_{\mathrm{c}}$, in quantifying a clustered earthquake sequence suggests that this assumption may be in error. Matthews et al. (2002) uses a Brownian passage-time distribution to construct an earthquake probability model. A seismic hazard analysis of southern California (Jackson et al. 1995) assumes a log-normal distribution of earthquake recurrence times. Abaimov et al. (2007) compares empirical earthquake distributions to only unimodal distributions. These and other analyses may have to be reconsidered in light of the possibility of a bimodal distribution of interseismic times for clustered earthquake sequences.

$B_{\text {c }}$ does have a shortcoming in that it does not take into consideration the relative sizes of the modes. One could imagine an earthquake sequence with a small number of short interseismic intervals and a large number of long ones. This would be considered clustered by our example (Fig. 5a), but examination of a small portion of the earthquake sequence itself would indicate a quasi-periodic sequence (Fig. 5b). Only the entire sequence is likely to reveal any earthquake clusters, as they are rare in this example. While this example may seem pathological (but not impossible), it does demonstrate an insensitivity of $B_{\mathrm{c}}$ to number of earthquake clusters. $B_{\mathrm{c}}$ instead mea- sures the difference in characteristic intra and intercluster seismic time intervals.

\section{RESULTS}

\subsection{Two-layer model}

Our reformulated definition of $W$ is

$W=\frac{\sigma_{\Delta \tau}}{\dot{\epsilon_{o}} \eta}=N W_{\mathrm{KS} 05}$,

where $\sigma_{\Delta \tau}$ is the standard deviation of the fault yield stress over the earthquake sequence, and $N=\sigma_{\Delta \tau} / \overline{\Delta \tau}$ is the fraction of noise added to the fault yield stress. We ran models with different rheological parameters, average and standard deviation of fault yield stress, and tectonic loading rates to determine the Wallace numberthat is, the single non-dimensional number that controls the degree of clustering for the system - for a 2-D lithospheric model with a faulted elastic layer overlying a single viscoelastic layer (Fig. 6).

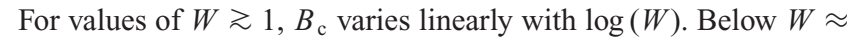
1 there is no temporal clustering: the distribution of the logarithm of interseismic intervals is normal (reflecting the distribution of the fault yield stress) and $B_{\mathrm{c}}=0$.

As stated earlier, we chose the log-normal distribution for the fault yield stress to avoid yield stresses less than zero, but this specific distribution is not necessary to obtain the bimodal distribution of interseismic times. A yield stress distribution that is derived from the log-normal distribution with all values below the mean discarded produces a similar distribution of interseismic times for low Wallace number systems and a bimodal distribution of interseismic times for those with a large Wallace number (Fig. 7).

While the amount of temporal clustering as measured by $B_{\mathrm{c}}$ is completely determined by the value of the Wallace number, the particular distribution of interseismic intervals, $T^{\mathrm{eq}}$, is not. For models where $W \gtrsim 1$, changing $W$ by varying the viscosity of the system
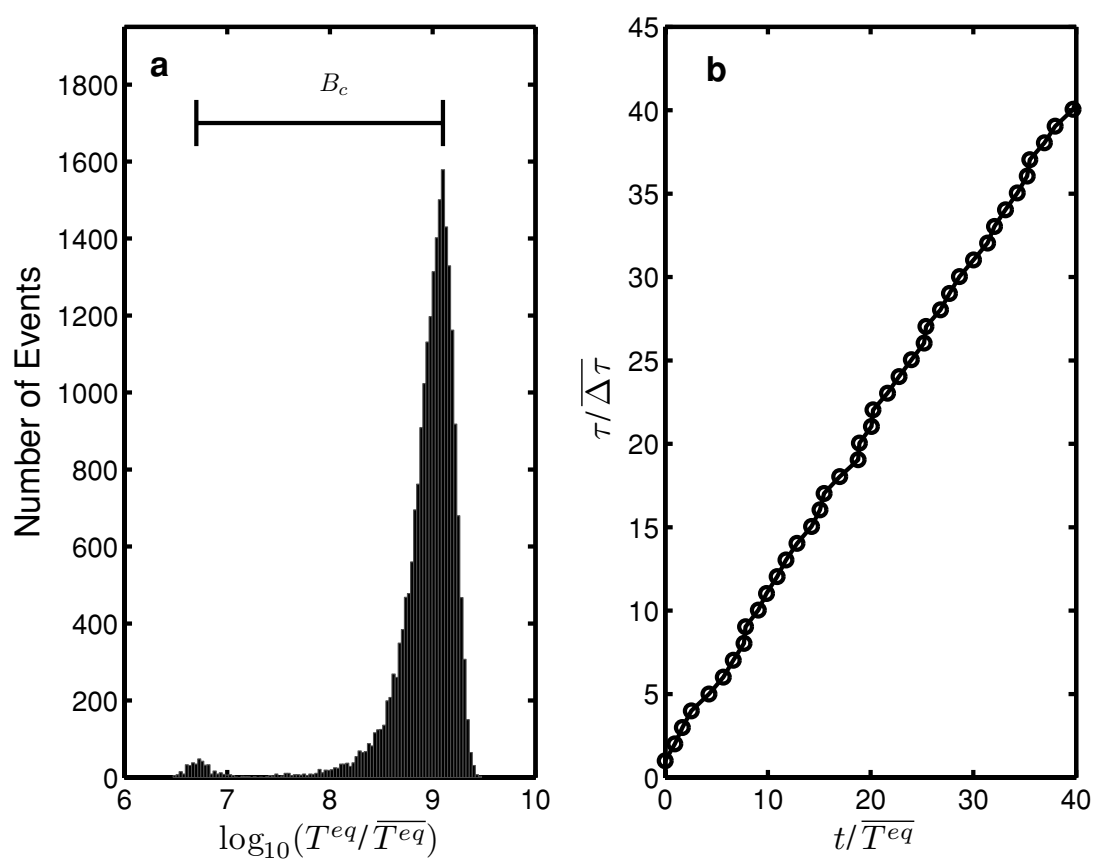

Figure 5. (a) Distribution of normalized interseismic intervals on a logarithmic scale for a model with $W=1.4 \times 10^{4}$ and $\sigma_{\Delta \tau} / \overline{\Delta \tau}=0.005$. (b) Cumulative stress released on the fault, $\tau$, normalized by the mean yield stress $\overline{\Delta \tau}$ versus normalized time. $\overline{T^{\mathrm{eq}}}$ is the mean interseismic interval. The earthquake time sequence appears quasi-periodic, but it is actually clustered by our measure. 

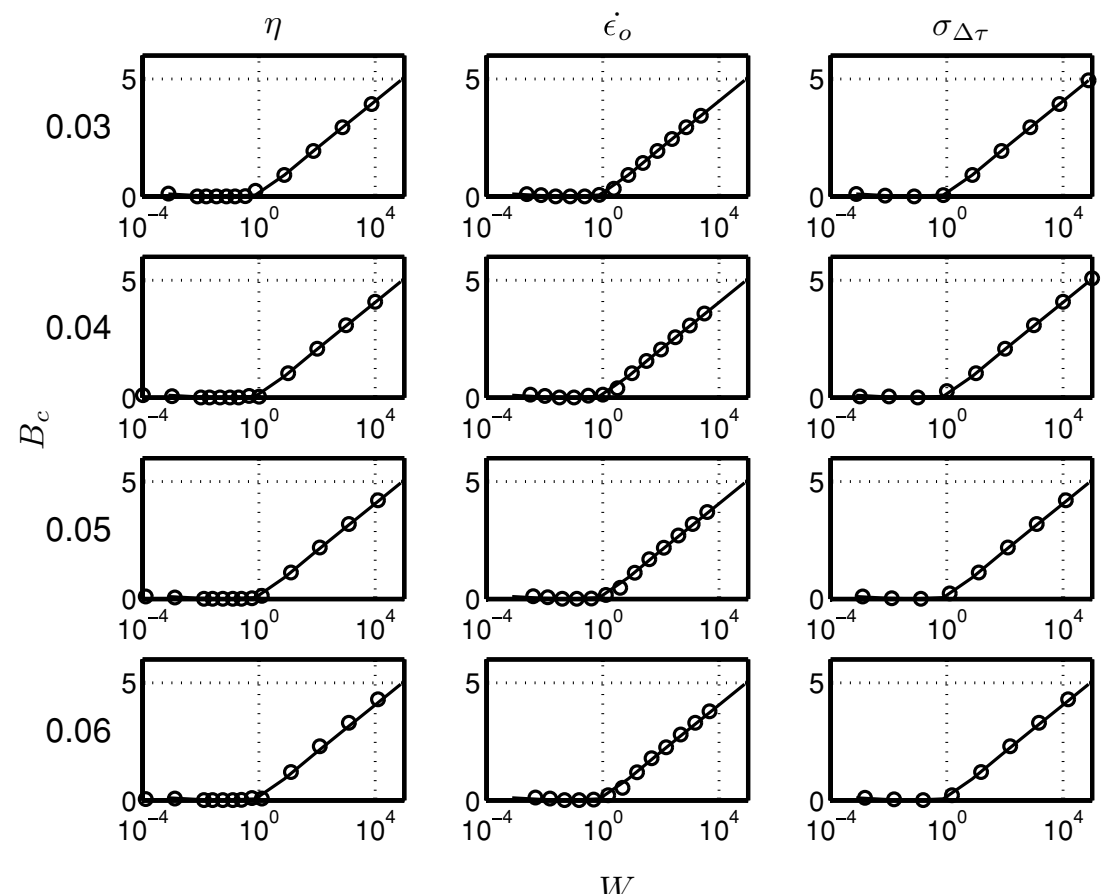

Figure 6. Amount of clustering in an earthquake sequence as measured by $B_{c}$ increases as the Wallace number, $W$, increases. Each row has a different value of $\mathrm{N}$ (the ratio of standard deviation to mean of the fault yield stress), and each column is a set of models changing a particular parameter of $W$. $\eta$ is the viscosity; $\dot{\epsilon}_{o}$ is the applied strain rate; and $\sigma_{\Delta \tau}$ is the standard deviation of the fault yield stress. The solid line is taken from the $N=0.03, \sigma_{\Delta \tau}$ plot and is the same in every panel.

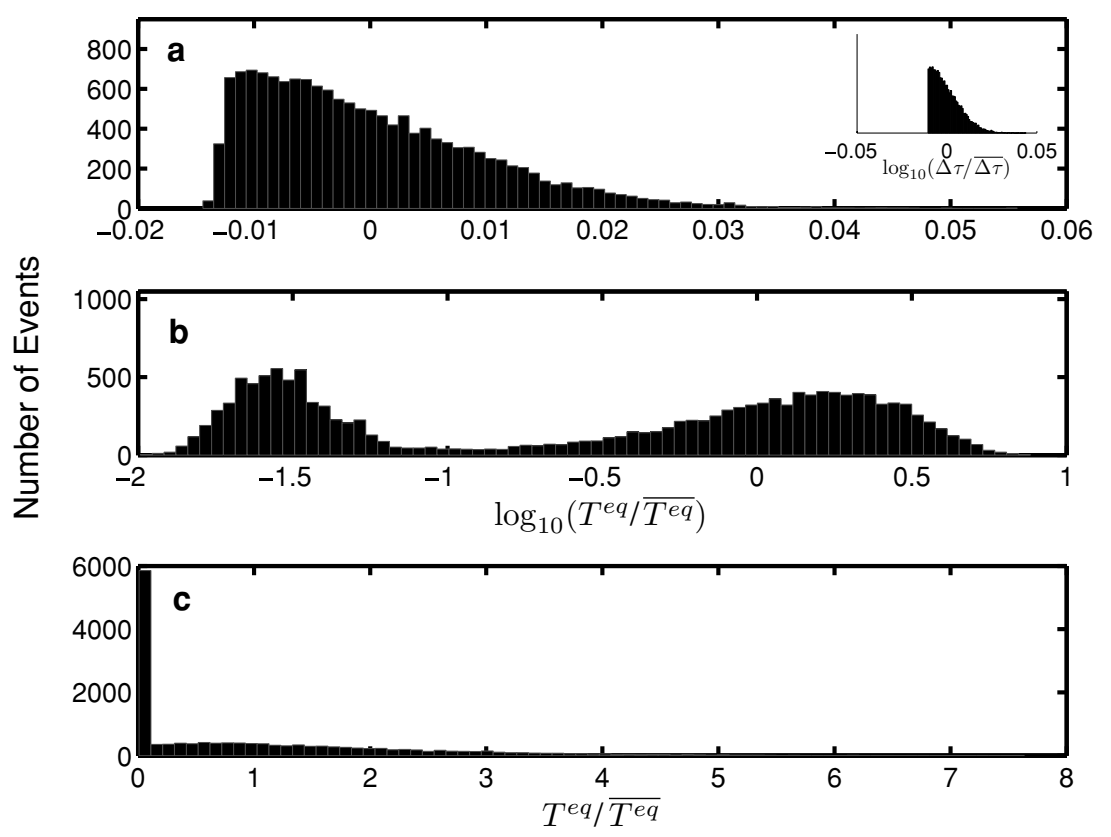

Figure 7. Same as Fig. 3 with different distribution of fault yield stress.

results in intracluster modes with nearly identical size and shape moving up the distribution of $\log \left(T^{\mathrm{eq}}\right)$ while the position, size, and shape of the intercluster is independent of $\eta$ (Fig. 8a). Of course, for the average interseismic interval, $\overline{T^{\mathrm{eq}}}$, to be constant from one model to the next (as it must be due to kinematic considerations) the modes cannot be absolutely identical, that is, a small, nearly undetectable change to the intercluster mode is all that is necessary to keep $\overline{T^{\mathrm{eq}}}$ constant. Similar behaviour is exhibited by groups of models with changing values of $\sigma_{\Delta \tau}$ and $\dot{\epsilon}_{o}$. However, in these cases, the intracluster mode remains stationary while the intercluster mode changes as $\sigma_{\Delta \tau}$ and $\dot{\epsilon}_{o}$ change (Figs $8 \mathrm{~b}$ and c).

The total number of earthquakes in each mode depends on the ratio $\sigma_{\Delta \tau} / \overline{\Delta \tau}$ (Fig. 9) in a linear fashion. The relative sizes of the modes does not change the amount of clustering as measured by $B_{\mathrm{c}}$ which quantifies the difference in interseismic interval during a clustered earthquake period and an intercluster period. 


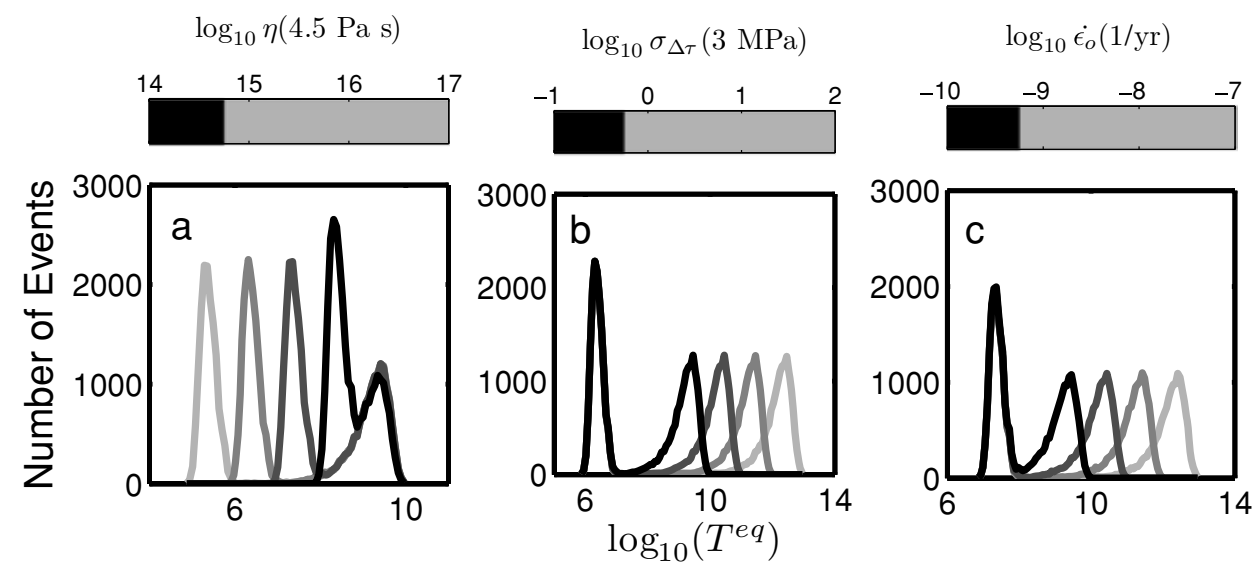

Figure 8. Distribution of normalized, logarithmic interseismic times for several values of $W$. In (a) the models have different values of viscosity, in (b) the models have different values of standard deviation of fault yield stress and in (c) the models have different values of the applied tectonic strain rate.

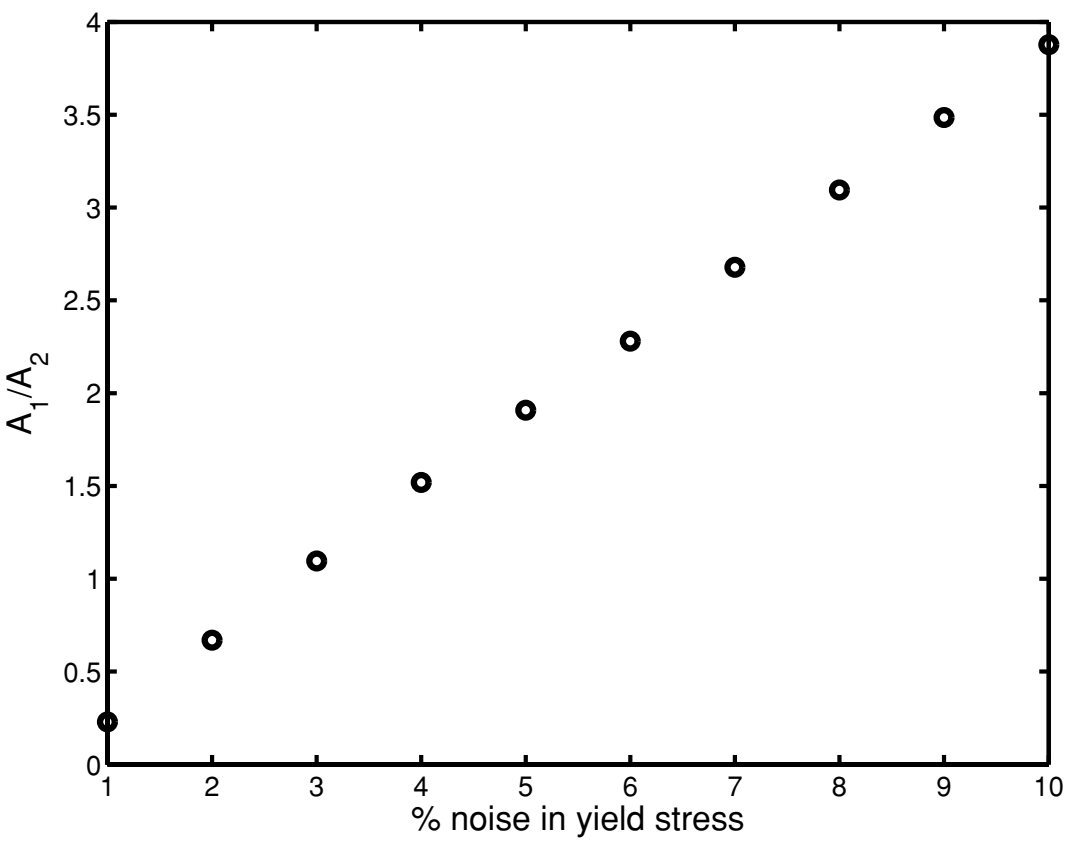

Figure 9. The ratio of earthquakes in the intracluster mode to the number of earthquakes in the intercluster mode as a function of the fraction of noise added to the fault yield stress.

\subsection{Three layer model}

The 1-D spring-dashpot-slider model used by KS05 has no inherent length scale; a system with multiple viscoelastic layers exhibits a single effective viscosity that is the arithmetic mean of all viscosities. In a horizontally layered, 2-D lithosphere, consisting of an elastic, seismogenic upper crust and viscoelastic layers below, viscoelastic layers not in direct contact with the elastic upper crust must communicate with it through the top-most viscoelastic layer. Therefore, we expect a more complicated scaling relationship between the model geometry and the viscosities of the individual layers determining the clustering behaviour of the system than that found by KS05.

We varied the relative thickness of layers two and three, while the total thickness of the model was held constant (Fig. 1). The viscosities of layers two and three are $4.5 \times 10^{15}$ and $4.5 \times 10^{17} \mathrm{~Pa} \mathrm{~s}$, respectively. The three-layer models exhibited similar bimodal clustering behaviour as the two-layer models (Fig. 10). The intracluster modes are broader and shorter than those of the two-layer mod- els. The value of $B_{\mathrm{c}}$ becomes smaller as the third layer (higher viscosity) is thickened. The upper viscoelastic layer, being in direct contact with the elastic upper crust has a stronger influence on the degree of clustering than does the lower viscoelastic layer. When $H_{2} / H_{3}=1.16$ the value of $B_{c}$ is nearly the same as the end-member model $\eta_{2}=\eta_{3}=4.5 \times 10^{15} \mathrm{Pas}$. When $H_{2} / H_{3}=0.06$ the value of $B_{\mathrm{c}}$ is close to halfway between the two-layer end member cases $\eta_{2}=\eta_{3}=4.5 \times 10^{15} \mathrm{Pas}$ and $\eta_{2}=\eta_{3}=4.5 \times 10^{17} \mathrm{Pas}$.

\section{DISCUSSION}

Our study using a 2-D, continuum FEM model finds that postseismic recycling of stress can cause earthquakes to cluster on a single fault when the yield stress on the fault is varied randomly from one earthquake to the next, confirming the conclusions of KS05 who used a 1-D analogue model for the viscoelastic lithosphere. Whether or not clustering occurs depends on the value of a non-dimensional number $W$; earthquake sequences are quasi-periodic for $W \lesssim 1$, and 


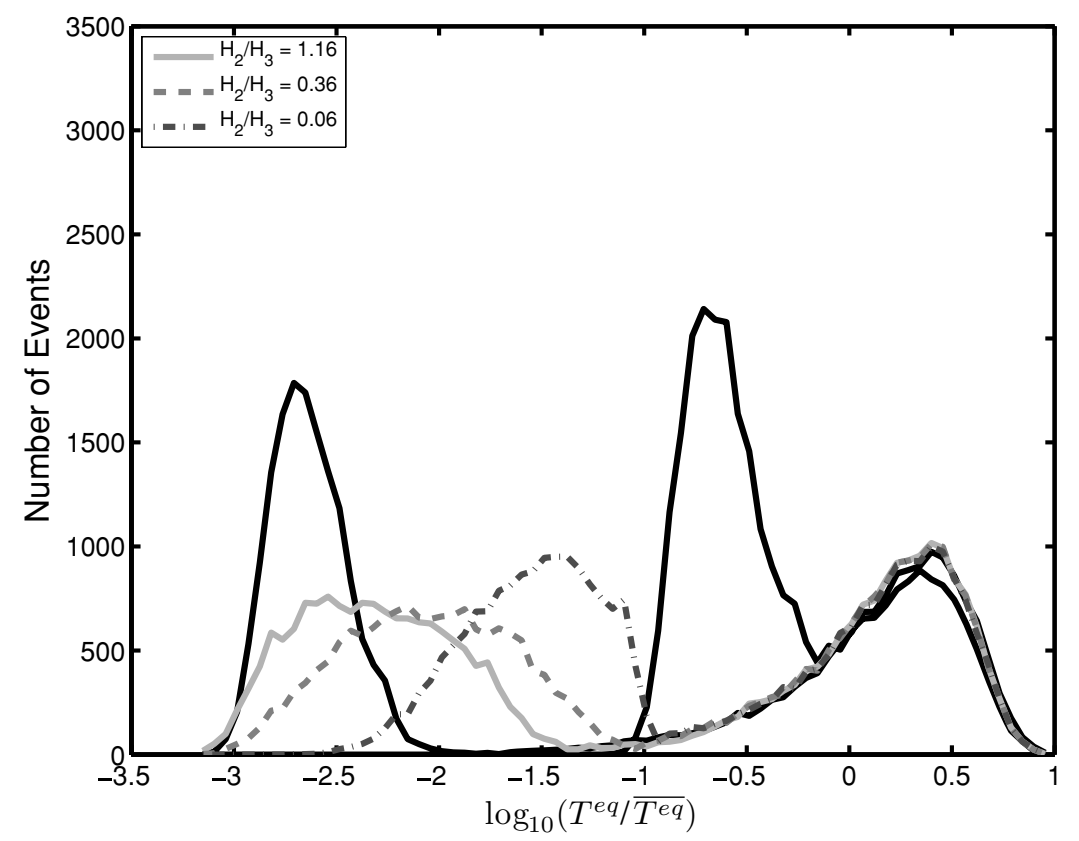

Figure 10. Distribution of normalized interseismic times in logarithmic space for models with an elastic layer over two viscoelastic layers (three-layer model). $\eta_{2}=4.5 \times 10^{15} \mathrm{Pas}$ and $\eta_{3}=4.5 \times 10^{17} \mathrm{Pas}$ (see Fig. 1 for model description). The total thickness of the viscoelastic layers is kept constant as the ratio of the layer thicknesses is changed. The black solid lines are the end-member cases of $\eta_{2}=\eta_{3}=4.5 \times 10^{15} \mathrm{Pas}$ (intracluster mode on the left) and $\eta_{2}=\eta_{3}=$ $4.5 \times 10^{17} \mathrm{~Pa}$ s (intracluster mode on the right).

clustered for larger values of $W$ by an amount depending linearly on $\log (W)$.

In order to compare the results from the 1-D model KS05 with that of a continuum model, we calculated $C_{v}$ and $W_{\mathrm{KS} 05}$ for models with $\sigma_{\Delta \tau} / \overline{\Delta \tau}=0.03-0.06$ and $W_{\mathrm{KS} 05}=2.5 \times 10^{-3}-2.5 \times 10^{5}$. A system is considered quasi-periodic when $C_{v} \approx \sigma_{\Delta \tau} / \overline{\Delta \tau}$, that is, the output earthquake sequence resembles the input yield stress (Fig. 3a). Values of $C_{v}>\sigma_{\Delta \tau} / \overline{\Delta \tau}$ are clustered, though the degree of clustering is difficult to ascertain because $C_{v}$ saturates for $W_{\mathrm{KS} 05} \gtrsim 1000$. The system transitions from quasi-periodic to clustered for values of $W_{\mathrm{KS} 05}$ between 1 and 100 (Fig. 4). These results are the same as those found by KS05. The general relationship between $W_{\mathrm{KS} 05}$ and $C_{v}$ is the same as in $\mathrm{KS} 05$, though the final values of $C_{v}$ are larger for our continuum models.

The 1-D spring-dashpot-slider model created by KS05 uses a coupling spring to transfer stress between rheological 'layers.' In addition to dependence on $W$, the clustered behaviour of the model depends on the ratio of the stiffness of the coupling spring to the stiffness of the top elastic 'layer' spring. Our 2-D continuum model of a strike-slip fault eliminates the need for the coupling spring due to the fact that the layers are inherently coupled.

We do not expect the averaging law for multiple viscoelastic layers to be a simple arithmetic mean as it is in the 1-D spring-dashpotslider analogue of KS05. Viscoelastic layers not in direct contact with the elastic upper crust must recycle their stress through the top-most viscoelastic layer, resulting in a complex relationship between the clustering behaviour of the system and the viscosities and thickness of the various layers. In addition, if a particular layer has a very large viscosity compared to the others it would no longer play a roll in the recycling of stress. We would expect that adding an extra elastic layer to the model would not change the rate of stress recycling except to the extent that it effects the geometry of the viscoelastic layers (Hetland \& Hager 2006b).

For a system with multiple viscoelastic layers there is more than one relaxation timescale, however there is only one phase of stress relaxation; there is one intracluster mode for models with two viscoelastic layers (Fig. 10). When the shear modulus is the same for all materials, as in the models discussed in this paper, the first timescale is simply the Maxwell relaxation time of the second layer. The second relaxation timescale however, is dependent on both the viscosities of the second layer and the third layer and is, therefore, not easily separated (Hetland \& Hager 2006b).

While the three layer model is not much more geologically realistic than the two-layer model, which is the primary focus of this paper, it does illuminate expected behaviour of more complex models such as those with multiple viscoelastic layers or a continuous grading of viscoelastic parameters with depth. Fault systems with multiple viscoelastic layers will have a single clustered mode that is broader and lower amplitude than the clustering mode for a system with only a single viscoelastic layer. The viscoelastic layer that is the closest to the elastic upper crust and the fault has the largest influence on the degree of clustering.

The average interseismic interval, $\overline{T^{\mathrm{eq}}}$, can be written in terms of components of $W$

$\overline{T^{\mathrm{eq}}}=\frac{\overline{\Delta \epsilon}}{\dot{\epsilon_{o}}}=\frac{\overline{\Delta \tau} / G}{\dot{\epsilon}_{o}}$

where $\overline{\Delta \epsilon}$ is the average coseismic strain drop and $G$ is the shear modulus of the elastic layer. The characteristic relaxation timescale for an elastic layer over a Maxwell viscoelastic half-space when the elastic parameters of the elastic layer and viscoelastic half-space are identical is (Hetland \& Hager 2005)

$T^{h s}=2 \frac{\eta}{G}$.

Combining eqs (3), (4), and (5) we get

$W=2 N \frac{\overline{T^{\mathrm{eq}}}}{T^{h s}}$. 


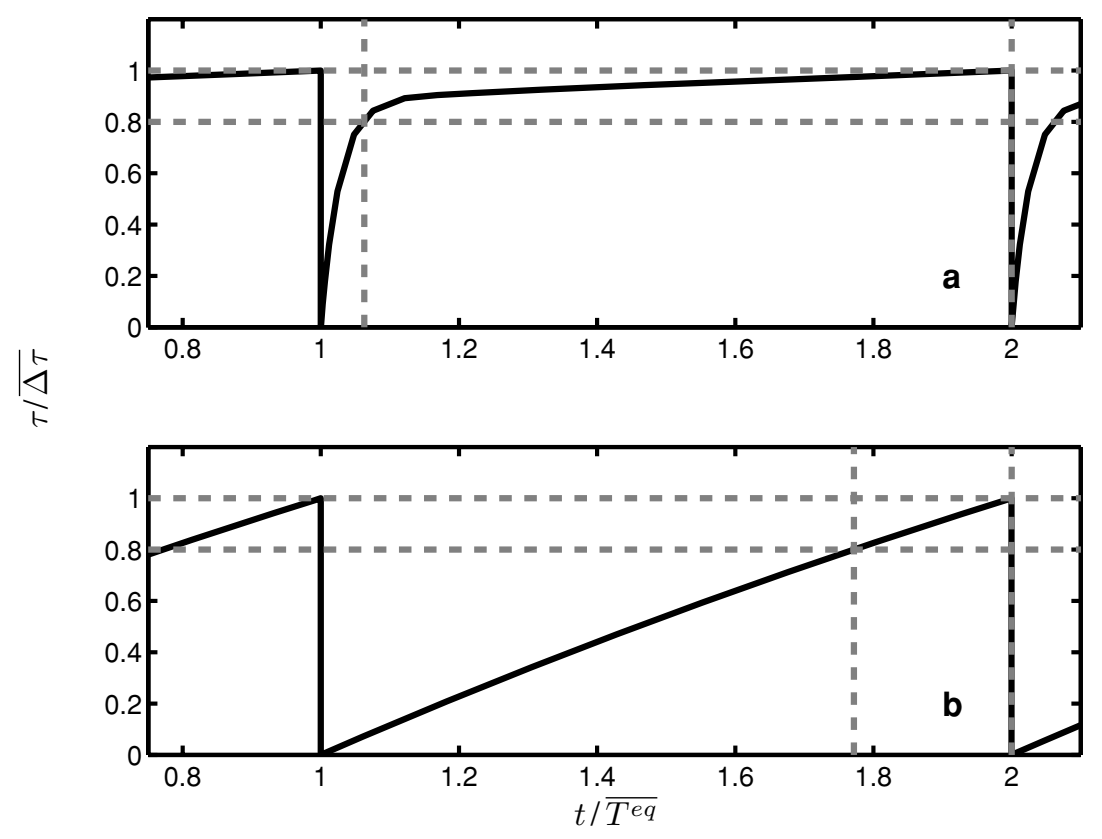

Figure 11. Average shear stress over the coseismic fault for two earthquake sequences. No noise has been added to the yield stress, so no clustering occurs. The dashed lines indicate the change in interseismic interval for a given change in yield stress. (a) When $W / N=85$, the shear stress rises rapidly early in the interseismic period due to rapid viscoelastic relaxation (relative to the tectonic loading rate). The loading rate then slows as the viscoelastic relaxation in the lower crust and the mantle slows. This non-linear loading results in sensitivity to changes in the yield stress from one earthquake to the next. (b) When $W / N=$ 0.85 the shear stress approaches the yield stress almost linearly throughout the entire earthquake sequences. Changes in the yield stress lead to approximately proportional changes in the interseismic time.

In terms of reloading rates

$W=2 N \frac{r^{h s}}{r^{o}}$

where $r^{h \mathrm{~s}}=1 / T^{h \mathrm{~s}}$ is the post-seismic viscoelastic reloading rate of our two-layer model (assuming it approximates an elastic layer over a viscoelastic half-space) and $r^{o}=1 / \overline{T^{\mathrm{eq}}}$ is the tectonic loading rate. Thus the Wallace number is a function of the amount of noise applied to the yield stress and the ratio of the tectonic loading rate to the viscoelastic relaxation loading rate. A system with a large $r^{h \mathrm{~s}} / r^{o}$ is sensitive to perturbations in the fault yield stress. The system moves close to failure soon after an earthquake due to rapid post-seismic relaxation; the fault loading then slows down to a rate much lower than the average loading rate (Fig. 11a). If the yield stress increases from the previous earthquake, the interseismic time becomes much longer due to the slow reloading at the end of the earthquake cycle. Conversely, if the yield stress decreases, then another earthquake occurs very quickly because the yield stress is reached during the rapid reloading phase. On the other hand, a system with a low $r^{h \mathrm{~s}} / r^{o}$ will not be as sensitive to changes in the fault yield stress. Because the fault is reloaded at a nearly constant rate (Fig. 11b), small changes in the fault yield stress result in small changes in the interseismic time - the distribution of interseismic intervals reflects the distribution of yield stresses (Fig. 3a).

Chery et al. (2001) also showed that post-seismic relaxation can influence the timing of earthquakes. A spring-dashpot-slider fault model is perturbed by the post-seismic viscoelastic deformation due to a neighbouring, parallel strike-slip fault, $150 \mathrm{~km}$ away. The coseismic stress drop on the fault is varied randomly from one earthquake to the next. Earthquake sequences on the two faults are clustered when the ratio of the average interseismic interval to the relaxation timescale is large. This ratio is the same as $W$ (eq. 7). Lynch et al. (2003) performed a similar study of fault interaction and clustering using a continuum model. They constructed a 3-D finite element model of the San Andreas fault with two seismogenic fault segments separated by a freely slipping, aseismic section. A seismogenic fault segment slipped when it exceeded a given yield stress. It was found that when the viscosity of the lower crust was small the two faults became coupled leading to clustered earthquake sequences.

Meade \& Hager (2004) noted that $W_{\mathrm{KS} 05}$ is the same as the Savage parameter: the ratio of average interseimic interval to the Maxwell viscoelastic relaxation timescale (Savage \& Prescott 1978); Hetland \& Hager (2006a) found $W_{\mathrm{Ks} 05}$ and the Savage parameter to differ by a factor of 4 . Meade \& Hager (2004) superposed the analytical solutions for surface displacements of an earthquake cycle for a strike-slip fault in an elastic layer over a Maxwell viscoelastic halfspace to analyse surface deformation for a clustered earthquake cycle. Systems with large values of the Savage parameter have a large range of surface velocities throughout the seismic cycle just as there is a large variability in the shear stress on the fault throughout the seismic cycle for our models with large $W / N=W_{\mathrm{Ks} 05}$ (Fig. 11).

One could view the viscoelastic system as a stress reservoir. We expect a cluster of earthquakes to occur when there is a large decrease in the fault yield stress. There is then a large reservoir of stress in the viscoelastic layers that causes rapid post-seismic relaxation for more than one earthquake. That reservoir of stress is fed back to the seismogenic fault at a rate faster for larger values of $W$; while for small values of $W$, the stress reservoir relaxes too slowly to cause clustering. As long as the yield stress remains low after the initial decrease, a large cluster will likely occur. Notice the cluster at $t / \overline{T^{\mathrm{eq}}}=63$ in Fig. 12: the cluster continues while the yield stress remains lower than that for the initial earthquake that started the cluster; the cluster ends when the reservoir of stress from the first earthquake cannot continue to drive the cluster in the face of an increase in yield stress. The increase in yield stress after the cluster is the same magnitude as a change early in the cluster: 


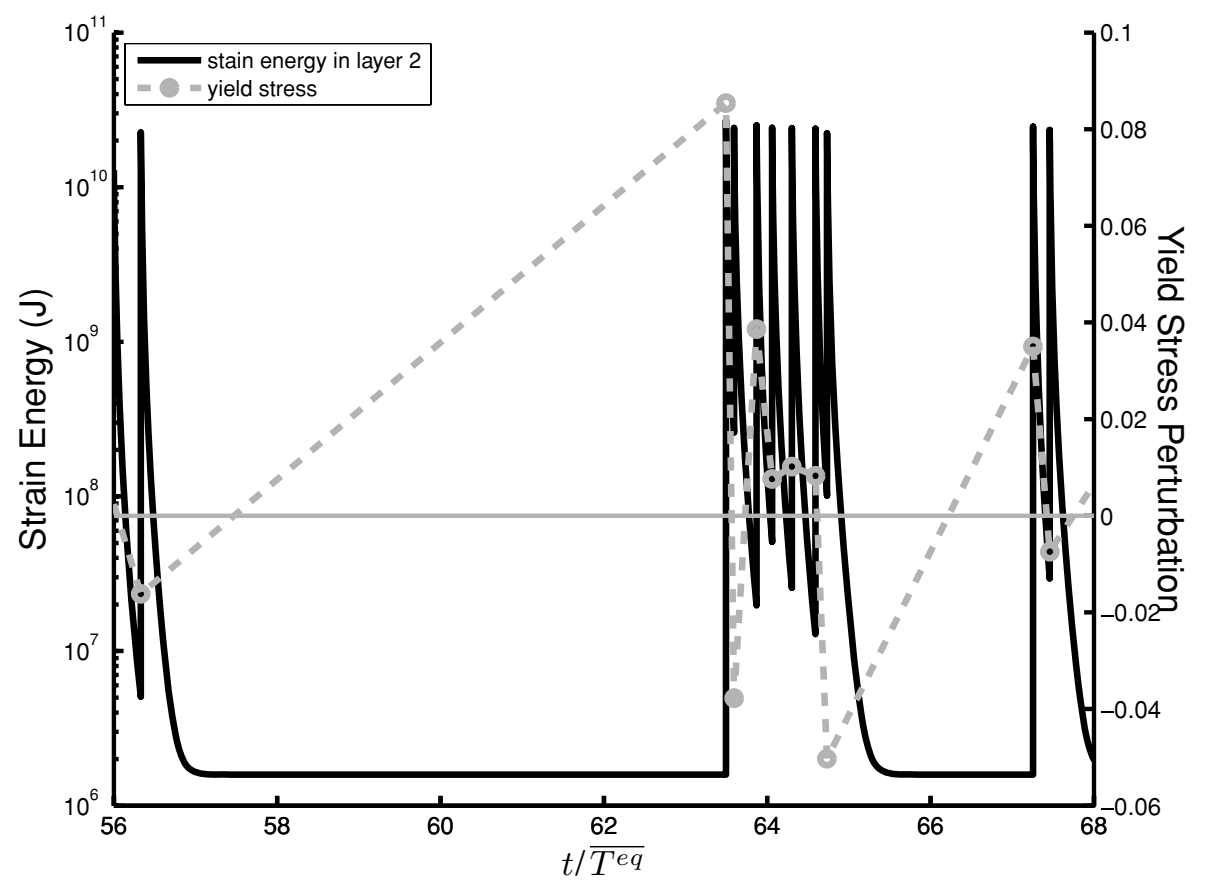

Figure 12. Strain energy (solid line) in the viscoelastic layer and the yield stress perturbation for a representative time period in a two-layer model with $\sigma_{\Delta \tau} / \overline{\Delta \tau}=0.03$ and $W=7.6$. An earthquake cluster occurs when a reservoir of stress in the viscoelastic layer due to a large stress drop continues to drive the reloading of the fault. Long intercluster periods occur when the reservoir of stress is exhausted and the yield stress increases.

the viscoelastic reloading is no longer rapid enough to continue the cluster.

Given a particular model geometry and viscosity, the system will evolve to a constant state of background stress, $\tau_{f}$, when the fault yield stress, $\Delta \tau$ is constant ( $\mathrm{KS} 05$ ). If the fault yield stress changes to $\Delta \tau^{\prime}$, the system begins to move to a new state of background stress, $\tau_{f}^{\prime}$. Hetland \& Hager (2006a) show that $\partial \tau / \partial \Delta \tau \sim W^{-1}$, where $\partial \tau=\tau_{f}-\tau_{f}^{\prime}$ and $\partial \Delta \tau=\Delta \tau-\Delta \tau^{\prime}$. For small values of $W$, changes in the fault yield stress will result in large changes in the overall state of stress of the system, meaning that deviations from a periodic rupture sequence are not likely. However, when $W$ is large, the deviations in yield stress produce negligible changes in the overall state of stress of the system making clustered sequences possible.

Ignoring past earthquakes (which we can do when $W$ is large, or equivalently the relaxation time is short), the stress on the fault during the interseismic period can be expressed as

$\tau(t)=\left(1-e^{t / T^{h s}}\right) \tau^{V E}+t r^{o}$,

where $\tau^{V E}$ is the maximum amount of stress recycled from the viscoelastic layer(s) and $r^{o}$ is the tectonic loading rate. For large $W$, the viscoelastic reloading occurs quickly relative to the tectonic loading; we can, therefore, consider the interseismic cycle to occur in two parts: a reloading phase and a tectonic phase. This two-phase interseismic period results in a significant asymmetry in the reloading curve. If, for a particular earthquake, the yield stress decreases significantly enough from the mean, then the earthquake will occur during the reloading phase of the interseismic cycle, and the tectonic term becomes negligible. We can express the earthquake stress drop — which is the same as the yield stress in our models — at the interseismic interval time $t=T^{\mathrm{eq}}$ as

$$
\Delta \tau\left(T^{\mathrm{eq}}\right)=\left(1-e^{T^{\mathrm{eq}} / T^{h s}}\right) \tau^{V E} .
$$

We rearrange terms to get

$$
T^{\mathrm{eq}}=-T^{h s} \ln \left(1-\frac{\Delta \tau}{\tau^{V E}}\right)=-2 \frac{\eta}{G} \ln \left(1-\frac{\Delta \tau}{\tau^{V E}}\right) .
$$

There is a linear relationship between $T^{h s}=2 \eta / G$ and the interseismic interval. This explains the slope of one for $W \gtrsim 1$ for the set of models with changing $\eta$ in Fig. 6. As $\eta$ increases, the intracluster mode moves to shorter $T^{\mathrm{eq}}$ while the intercluster mode is relatively stationary (Fig. 8). When $\Delta \tau$ increases from the average, the corresponding increase in $T^{\mathrm{eq}}$ is nearly independent of $\eta$. Fig. 13 shows the asymmetry of the reloading curve and the viscosity dependence of the intracluster earthquake times. For $W \lesssim 1$, the tectonic term becomes more important in the analysis and the reloading curve becomes more symmetric, resulting in non-clustered, quasi-periodic behaviour. We can use a similar logic to explain the inverse relationship between the degree of clustering and the applied tectonic strain rate and the standard deviation of the earthquake yield stress.

Of course, if $\Delta \tau$ is smaller than $\overline{\Delta \tau}$ for several earthquakes in a row, then the assumption that we can ignore the effect of viscoelastic relaxation from past earthquakes no longer holds. The loading of the fault due to viscoelastic relaxation happens even faster and a long cluster of earthquakes takes place as the reservoir of stress stored in the viscoelastic layers is recycled back to the seismogenic crust.

A model studied by Ben-Zion et al. (1993) found that the viscoelastic post-seismic relaxation from a kinematically imposed large earthquake on the southern portion of the San Andreas fault could cause the nearby regions to have clustered earthquakes soon after the large event and fewer earthquakes later in the large earthquake cycle. This change in seismic rate is due to the large changes in postseismic loading rates from a low viscosity lower crust. Specifically, small values of the ratio of viscous relaxation time to lower crustal thickness cause clustering. 


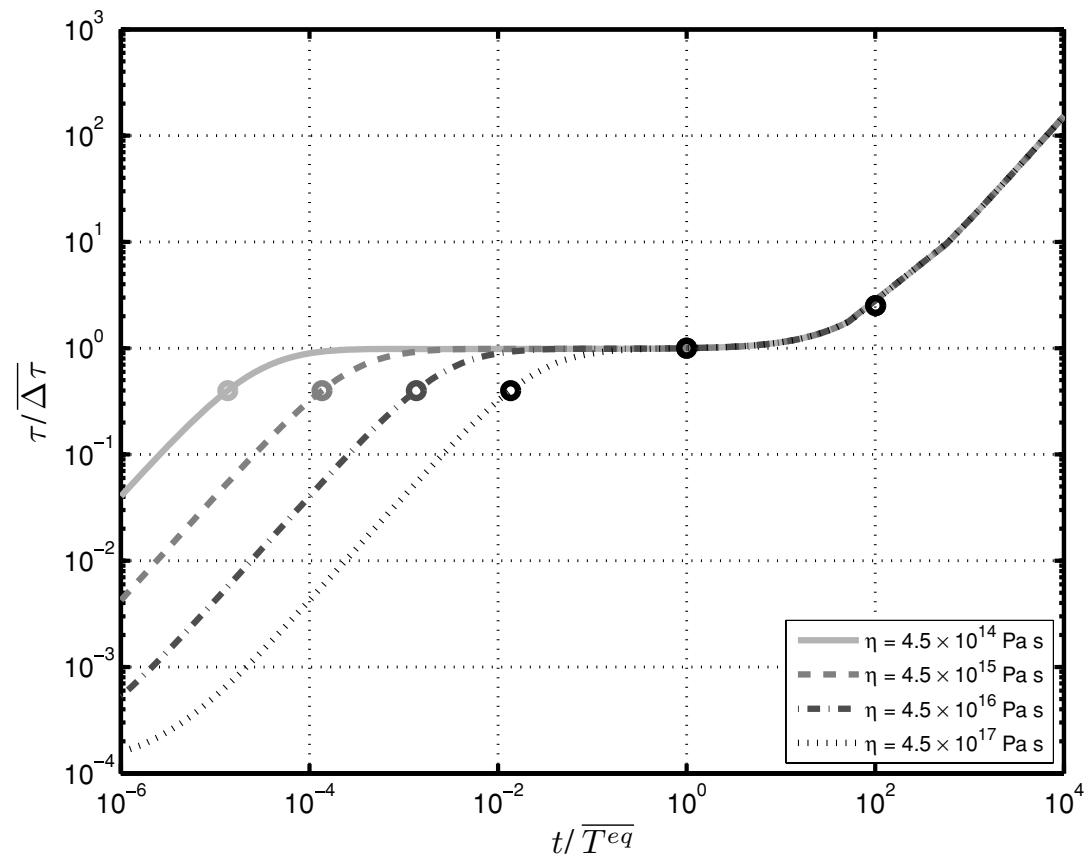

Figure 13. Average shear stress over coseismic fault as a function of time. The circles are at $\overline{\Delta \tau}$ and $\overline{\Delta \tau} \pm \sigma_{\Delta \tau}$. For systems with a large Wallace number, reloading comes in two phases: a viscoelastic rebound phase and a tectonic loading phase. The average fault yield stress falls on the flat portion of the reloading curve, while clustered periods occur during the viscoelastic rebound phase and intercluster periods are in the tectonic loading phase.

\section{CONCLUSIONS}

Using a continuum, finite element approach we studied the temporal clustering of characteristic earthquakes on a single fault. We modelled an infinitely long strike-slip fault using an antiplane 2-D geometry. To best identify clustering behaviour we considered histograms of interseismic times in logarithmic space. In this space, two modes clearly appear, an intracluster interval mode and an intercluster interval mode; we define a clustering metric, $B_{\mathrm{c}}$, as the distance between these modes. Clustering is triggered by random perturbations in the fault yield stress from one earthquake to the next and occurs when the Wallace number-which is a function of the amount of noise applied to the system and the ratio of the tectonic loading rate to the viscoelastic relaxation loading rate (eq. 7)-is larger then 1 . The degree of clustering as measured by $B_{\mathrm{c}}$ is linearly related to the log of the Wallace number.

In real geological settings we expect the viscosity structure to be more complex than those studied in this model. The temperature gradient in the mantle and the expected temperature dependence of viscosity (e.g. Ranalli 1991) suggests that the viscosity gradient over the lithosphere is significant. In this case, we would expect to see a broad distribution of interseismic intervals for a temporally clustered earthquake mode. However, the rheology near the elastic upper crust has the strongest influence on the degree of clustering.

Comparison to real fault systems is difficult because of the many complexities found in real geological settings. Aside from the problem of rheological structure mentioned above, there is the complication of fault geometry and, more importantly, interactions from other faults. Fault interactions can come in the form of strain partitioning (requiring strain rates to be estimated for a specific fault rather than a geological region) and in fault communication through postseismic processes as previously addressed by Chery et al. (2001) and Lynch et al. (2003). The models presented here are used to study the interaction between post-seismic processes, fault load- ing, and temporal earthquake clustering with as few complexities as possible in order to build an intuitive understanding of the processes at hand. Having said that, it is possible to estimate values of $W / N$ for various tectonic settings to qualitatively find the likelihood of earthquake clustering due to post-seismic viscoelastic deformation.

Typical values of earthquake stress drop are 1-10 MPa (Kanamori \& Anderson 1975). The velocity across the San Andreas fault has been measured geodetically as about $35 \mathrm{~mm} \mathrm{yr}^{-1}$ (e.g. Prescott et al. 2001). The Maxwell viscosity is in the range $\eta=10^{19}-10^{20} \mathrm{Pas}$ (e.g. Thatcher 1983; Li \& Rice 1987; Kenner \& Segall 2000). If we assume $\dot{\epsilon}_{o}=v / d$, where $d \approx 15 \mathrm{~km}$ is the thickness of the seismogenic crust, then we get $W / N \approx 0.1-14$. In contrast, the Dead Sea transform has a much lower slip rate and is thought to have a low viscosity lower crust. The velocity across the fault has been measured geologically as 2-6 $\mathrm{mm} \mathrm{yr}^{-1}$ (Klinger et al. 2000); viscosities are in the range $5 \times 10^{17}-5 \times 10^{19} \mathrm{Pas}$ (Al-Zoubi \& ten Brink 2002), which gives $W / N=1-5000$. At a value of $N$ of 3 per cent we would expect that post-seismic, viscoelastic relaxation could play a role in creating temporally clustered earthquake sequences on the Dead Sea transform but not the San Andreas fault. The effects of non-linear viscosities complicate the problem; we would expect the recycling of stress leading to clustered behaviour to be even stronger in this case as the viscosity decreases when the viscoelastic layers are coseismically stressed increasing the effective Wallace number.

Our results from a two-layer, single fault, finite element model agree with those found by KS05 using a 1-D, spring-dashpot-slider analogue. These results are also consistent with the findings of Lynch et al. (2003) and Chery et al. (2001) who studied viscoelastic relaxation and fault interaction. The clustering behaviour exhibited in this study is expected to occur in any lithosphere model with time dependent rheology; that is, a rheology that is sensitive to the earthquake history (e.g. Ben-Zion et al. 1999; Chery et al. 2001; Lyakhovsky et al. 2001; Lynch et al. 2003; Chery \& Vernant 2006). 


\section{ACKNOWLEDGMENTS}

We thank Eric Hetland for insightful discussions on this work. We also acknowledge careful reviews from two anonymous reviewers and Dr M. Cocco. This work was partially funded by NSF grant EAR-0229868. Caltech Seismological Laboratory contribution 9169 .

\section{REFER E N CES}

Abaimov, S.G., Turcotte, D.L. \& Rundle, J.B., 2007. Recurrence-time and frequency-slip statistics of slip events on the creeping section of the San Andreas fault in central California, Geophys. J. Int., doi: 10.1111/j.1365246X.2007.03479.x.

Al-Zoubi, A. \& ten Brink, U., 2002. Lower crustal flow and the role of shear in basin subsidence: an example from the Dead Sea basin, Earth planet. Sci. Lett., 199(1-2), 67-79.

Begin, Z., Steinberg, D., Ichinose, G. \& Marco, S., 2005. A 40,000 year unchanging seismic regime in the Dead Sea rift, Geology, 33, 257-260.

Ben-Zion, Y., Dahmen, K., Lyakhovsky, V., Ertas, D. \& Agnon, A., 1999. Self-driven mode switching of earthquake activity on a fault system, Earth planet. Sci. Lett., 172, 11-21.

Ben-Zion, Y., Rice, J. \& Dmowska, R., 1993. Interaction of the San Andreas fault creeping segment with adjacent great rupture zones and earthquake recurrence at Parkfield, J. geophys. Res., 98, 2135-2144.

Chandrasekharaiah, D. \& Debnath, L., 1994. Continuum Mehanics, Academic Press, San Diego, CA.

Chery, J. \& Vernant, P., 2006. Lithospheric elasticity promotes episodic fault activity, Earth planet. Sci. Lett., 243(1-2), 211-217.

Chery, J., Merkel, S. \& Bouissou, S., 2001. A physical basis for time clustering of large earthquakes, Bull. seism. Soc. Am., 91, 1685-1693.

Friedrich, A., Wernicke, B., Niemi, N., Bennett, R. \& Davis, J., 2003. Comparison of geodetic and geologic data from the Wasatch region, Utah, and implications for the spectral character of Earth deformation at periods of 10 to 10 million years, J. geophys. Res., 108, 2199.

Grant, L. \& Sieh, K., 1994. Paleoseismic evidence of clustered earthquakes on the San Andreas fault in the Carrizo Plain, California, J. geophys. Res., 99, 6819-6841.

Hetland, E. \& Hager, B., 2005. Postseismic and interseismic displacements near a strike-slip fault: a two-dimensional theory for general linear viscoelastic rheologies, J. geophys. Res., 110, B10401.

Hetland, E.A. \& Hager, B.H., 2006a. Interseismic strain accumulation: spinup, cycle invariance, and irregular rupture sequences, Geochem. Geophys. Geosyst., 7, Q05004.

Hetland, E.A. \& Hager, B.H., 2006b. The effects of rheological layering on post-seismic deformation, Geophys. J. Int., 166(1), 277-292.

Jackson, D.D., Aki, K., Cornell, C.A., Dieterich, J.H., Henyey, T.L., Mahdyiar, M., Schwartz, D. \& Ward, S.N., 1995. Seismic hazards in southern California-probable earthquakes, 1994 to 2024, Bull. seism. Soc. Am., 85(2), 379-439.

Kagan, Y. \& Jackson, D., 1991. Long-term earthquake clustering, Geophys. J. Int., 104, 117-133.
Kanamori, H. \& Anderson, D.L., 1975. Theoretical basis of some empirical relations in seismology, Bull. seism. Soc. Am., 65(5), 1073-1095.

Kenner, S. \& Segall, P., 2000. Postseismic deformation following the 1906 San Francisco earthquake, J. geophys. Res., 105, 13195-13209.

Kenner, S. \& Simons, M., 2005. Temporal clustering of major earthquakes along individual faults due to post-seismic reloading, Geophys. J. Int., 160, 179-194.

Klinger, Y., Avouac, J.P., Abou Karaki, N., Dorbath, L., Bourles, D. \& Reyss, J.L., 2000. Slip rate on the Dead Sea transform fault in northern Araba valley (Jordan), Geophys. J. Int., 142(3), 755-768.

Li, V. \& Rice, J., 1987. Crustal deformation in great California earthquake cycles, J. geophys. Res., 92, 11533-11551.

Lyakhovsky, V., Ben-Zion, Y. \& Agnon, A., 2001. Earthquake cycle, fault zones, and seismicity patterns in a theologically layered lithosphere, $J$. geophys. Res., 106, 4103-4120.

Lynch, J., Burgmann, R., Richards, M. \& Ferencz, R., 2003. When faults communicate: viscoelastic coupling and earthquake clustering in a simple two-fault system, Geophys. Res. Lett., 30, 1270.

Marco, S., Stein, M., Agnon, A. \& Ron, H., 1996. Long-term earthquake clustering: a 50,000-year paleoseismic record in the Dead Sea graben, $J$. geophys. Res., 101, 6179-6191.

Matthews, M.V., Ellsworth, W.L. \& Reasenberg, P.A., 2002. A brownian model for recurrent earthquakes, Bull. seism. Soc. Am., 92(6), 2233-2250.

Meade, B. \& Hager, B., 2004. Viscoelastic deformation for a clustered earthquake cycle, Geophys. Res. Lett., 31, L10610.

Melosh, H. \& Raefsky, A., 1980. Dynamical origin of subduction zone topography, Geophys. J. Int., 60, 333-354.

Prescott, W.H., Savage, J.C., Svarc, J.L. \& Manaker, D., 2001. Deformation across the Pacific-North America plate boundary near San Francisco, California, J. geophys. Res., 106(B4), 6673-6682.

Ranalli, G., 1991. The microphysical approach to mantle rheology, in Glacial Isostasy, Sea-Level and Mantle Rheology, pp. 493-513, eds Sabadini, R., Lambeck, K. \& Boschi, E., Kluwer Academic Publishers, Boston, MA.

Savage, J.C. \& Prescott, W.H., 1978. Asthenosphere readjustment and earthquake cycle, J. geophys. Res., 83(NB7), 3369-3376.

Schwartz, D. \& Coppersmith, K., 1984. Fault behavior and characteristic earthquakes - examples from the Wasatch and San Andreas fault zones, J. geophys. Res., 89, 5681-5698.

Tarantola, 2006. Elements for Physics: Quantities, Qualities, and Intrinsic Theories, Springer Berlin.

Thatcher, W., 1983. Non-linear strain buildup and the earthquake cycle on the San Andreas fault, J. geophys. Res., 88(NB7), 5893-5902.

Wallace, R., 1987. Grouping and migration of surface faulting and variations in slip rates on faults in the Great Basin province, Bull. seism. Soc. Am., 77, 868-876.

Weldon, R.J., Scharer, K.M., Fumal, T.E. \& Biasi, G.P., 2004. Wrightwood and the earthquake cycle: what a long recurrence record tells us about how faults work, GSA Today, 14, 4-10.

Williams, C. \& Wadge, G., 2000. An accurate and efficient method for including the effects of topography in three-dimensional elastic models of ground deformation with applications to radar interferometry, J. geophys. Res., 105, 8103-8120. 